Témoigner Témoigner. Entre histoire et mémoire

Getuigen Revue pluridisciplinaire de la Fondation Auschwitz

$121 \mid 2015$

Violences radicales en scène

\title{
Le théâtre documentaire en Arménie
}

Modes de représentation du génocide dans les pièces de

Pertj Zeytountsyan

\section{Annick Asso}

\section{(2) OpenEdition}

1 Journals

\section{Édition électronique}

URL : https://journals.openedition.org/temoigner/3299

DOI : $10.4000 /$ temoigner.3299

ISSN : 2506-6390

Éditeur :

Éditions du Centre d'études et de documentation Mémoire d'Auschwitz, Éditions Kimé

Édition imprimée

Date de publication : 1 octobre 2015

Pagination : $69-75$

ISSN : 2031-4183

Référence électronique

Annick Asso, «Le théâtre documentaire en Arménie », Témoigner. Entre histoire et mémoire [En ligne],

121 | 2015, mis en ligne le 01 octobre 2016, consulté le 04 février 2022. URL : http://

journals.openedition.org/temoigner/3299; DOI : https://doi.org/10.4000/temoigner.3299

Tous droits réservés 


\title{
Le théâtre documentaire en Arménie
}

\author{
Modes de représentation du génocide \\ dans les pièces de Pertj Zeytountsyan
}

$\rightarrow$ Par Annick Asso,

Université de Montpellier III RIRRA 21

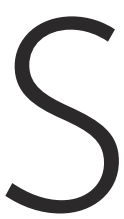

'inscrivant dans une longue tradition théâtrale arménienne, Pertj Zeytountsyan, né en 1938, est le dramaturge contemporain le plus joué en Arménie. Trois pièces de son répertoire, Le Grand Silence, Debout ! Le tribunal arrive, Cent ans après, traitent du génocide des Arméniens, trois pièces situées dans trois temporalités : pendant, en 1915, après, en 1921, aujourd'hui, cent ans après. Ces œuvres participent de la résurgence du théâtre documentaire en Arménie, au moment même où ce théâtre, hérité d'Erwin Piscator et Peter Weiss, réapparaît sous des formes similaires en Europe et en Russie. Pertj Zeytountsyan s'inspire du théâtre documentaire défini par Peter Weiss, un genre particulièrement adapté à la représentation des événements historiques tels que les guerres, les expéditions de pillages et les génocides, et il adjoint à cette forme une réflexion sur la mémoire collective constitutive de l'identité arménienne. Pour le dramaturge, le génocide s'est imposé comme un sujet théâtral à la fin de l'ère soviétique qui, pendant des décennies, avait érigé le discours et la représentation du génocide en tabou. Une difficulté était néanmoins de trouver la juste distance qui permette la représentation de cette réalité devant un public continuant d'éprouver les conséquences de l'événement, sur le plan géopolitique et sur le plan psychologique, en raison de la non-reconnaissance et de la négation du génocide par la Turquie, mais aussi du blocus que la Turquie continue d'exercer sur l'Arménie ainsi que de la récente guerre au Karabagh.

\section{SYNOPSIS}

Le Grand Silence (Medz Lerutjun), pièce représentée en 1990, est une reconstitution historique des derniers jours des poètes Rubèn Sevak et Daniel Varoujan, arrêtés dans la nuit du 24 avril 1915, déportés avec quelques centaines d'autres écrivains et intellectuels à la prison de Tchanguiri, au cœur de l'Anatolie, dans la région d'Ankara, où, après deux semaines d'emprisonnement, ils sont exécutés dans les pires souffrances. Avec Le Grand Silence, Pertj Zeytountsyan produit une pièce emblématique. En effet, la destruction de l'élite arménienne est systématique et à peu près totale : deux mille personnalités sont ainsi éliminées dès le mois d'avril 1915. L’objectif du 
Le théâtre documentaire en Arménie

(suite) gouvernement Jeunes-Turcs était de « couper la tête au peuple arménien avant d'en fracasser les membres. » (Lepsius, 1987, 30) Du point de vue de l'histoire littéraire arménienne, l'événement est également d'une signification primordiale dans la mesure où pendant des décennies, suite au génocide, l'élite intellectuelle arménienne peinera à se reconstituer, en Arménie, et encore plus en diaspora. La pièce porte donc directement sur ce point nodal, la date symbolique des commémorations du génocide, et nous transporte dans un temps-repère, en avril 1915, en nous plaçant dans l'état d'esprit de nombreux Arméniens de l'époque. Pertj Zeytountsyan fait de Daniel Varoujan un personnage contrasté, à la fois poète visionnaire et figure de l'optimisme aveuglé. Et de fait, à la veille du génocide, les Arméniens sont, d'un côté conscients des menaces qui pèsent sur eux, les massacres de Cilicie en 1909 sont annonciateurs du Medz Yeghern, de la grande Catastrophe qui s'ensuit. De l'autre côté, la population arménienne est dans une impossibilité presque viscérale d'envisager la réalisation du pire, à plus grande échelle. Comment les Arméniens peuvent-ils imaginer être chassés définitivement de leurs terres ancestrales, dans un Empire où ils cohabitent en liens étroits, au quotidien, avec les Turcs? Ils continuent, jusqu'au bout, à manifester leur confiance envers le nouveau régime Jeune-Turc dans lequel ils ont placé tant d'espoir.

Debout! Le tribunal arrive (Votk'i Datarann é galis) représente sur scène le procès du justicier du génocide, Soghomon Téhléryan, qui, dans le cadre de l'opération Némésis, assassine en 1921, à Berlin, le principal ordonnateur du génocide, le ministre de l'Intérieur Jeune-Turc, Talaat Pacha, condamné à mort par contumace par le tribunal militaire turc de 1919 et dont la sentence n’avait jamais été exécutée. La pièce nous introduit dans le temps du « juste après » le génocide, alors que les séquelles sont vives chez les rescapés représentés par la figure emblématique de Soghomon Téhléryan, un personnage qui se débat avec ses souvenirs terrifiants, devant une cour pénale étrangère où le crime de génocide n'est pas encore nommé. Ce que tend à montrer la pièce, c'est que son acte n'est pas un acte isolé, il est évidemment chargé de toute la souffrance d'un peuple en attente d'une reconnaissance sur la scène internationale. Cette pièce, dont la première représentation date de 1988, est la première qui porte sur le génocide, écrite et jouée pendant la période soviétique. Elle connaît un immense succès en Arménie : elle est représentée au Théâtre Sundukyan d'Erevan, chaque année, depuis le 16 septembre 2000, dans une mise en scène de Tigran Gasparyan.

Cent ans après (Hariuj dari anc) est la dernière pièce écrite par Pertj Zeytounstian et jouée actuellement au Théâtre Sundukyan, dans une mise en scène de Tigran Gasparyan, dans le cadre des commémorations du centenaire du génocide. La pièce s'inscrit dans le temps de l'après-génocide, cette fois, comme l'on serait tenté de le dire, longtemps après. Elle met en scène les descendants, un Turc et un Arménien. Pour l'argument de la pièce, Pertj Zeytountsian s'inspire de la venue à Erevan, en 2008, du journaliste turc Hassan Djemal, petit fils de Djemal Pacha, ministre de la Marine et ordonnateur du génocide de 1915 : Hassan Djemal, ami proche d'Hrant Dink, au terme d'un parcours journalistique, politique et personnel est venu deman- 


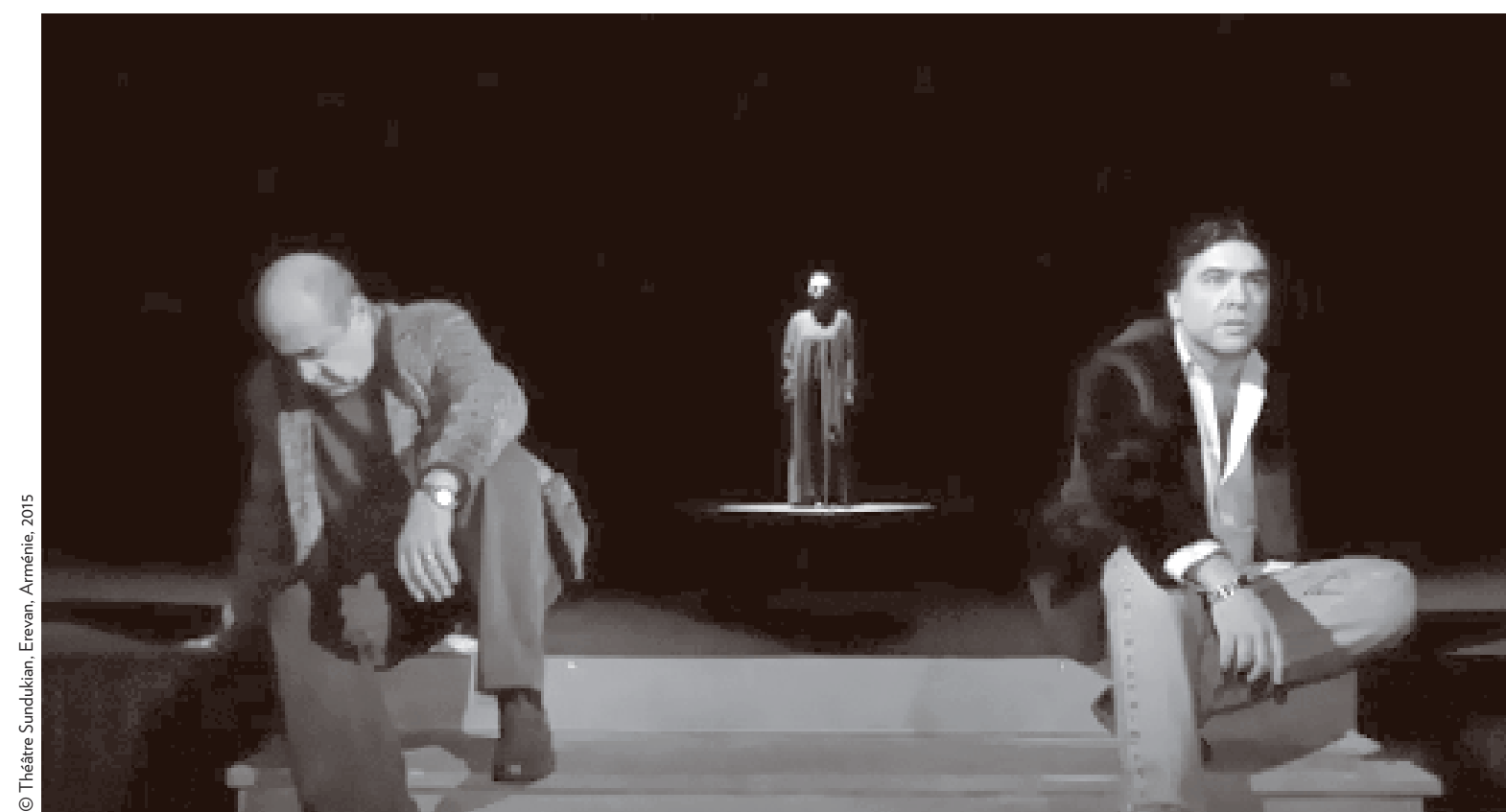

der pardon aux Arméniens et déposer un bouquet de roses blanches au Mémorial du génocide, en insistant sur le fait que le gouvernement turc devait également reconnaître le génocide. À Erevan, Hassan Djemal rencontre Armen Guevorkian, petit-fils du justicier Artachès Guevorkian qui, comme Soghomon Téhléryan, appartenait au commando Némésis et a assassiné son grand-père Djemal Pacha. Pertj Zeytountsyan transpose la rencontre entre ces deux petits-fils, en un duel agonistique où chacun se débat avec sa propre vérité bien plus que contre l'autre. Sur scène, les noms sont transposés : Hassan Djemal devient Kémal, et Armen Guévorkian se prénomme Antranik. Finalement, dans la pièce, après avoir surmonté ses contradictions internes, Kemal décide de se rendre au Mémorial Tzitsernakapert, à Erevan, et dépose les fleurs en mémoire des victimes du génocide « pour mon grand-père aussi, dit-il, pour moi, lui aussi est une victime... »

\section{INSERTION DU DOCUMENT \\ ET LA RÉÉCRITURE DU TÉMOIGNAGE}

Conformément à l'esthétique documentaire, Pertj Zytountsyan utilise un montage de documents destinés à retracer les faits historiques. Dans Le Grand Silence, les conditions de l'exécution des deux poètes sont clairement établies à la scène 5 , par l'insertion de documents de l'Ittihad. La scène retrace la confrontation des prisonniers avec leur bourreau, Djemal Oghuz, qui prépare leur exécution au moment
_À gauche, Kémal (Hassan Djemal, le petit fils de Djemal Pacha, ministre de la Marine et ordonnateur du génocide), au fond Mariam (L’apparition), à droite Andranik (Armen Guévorkian, le petit-fils d'Artachès Guévorkian, assassin de Djemal Pacha). Cent ans après de Pertj Zeytountsyan, mise en scène de Tigran Gasparya. 
Le théâtre documentaire en Arménie

(suite) du transfert prévu de Tchanguiri à Ayach, avec trois autres déportés. Ce sont ensuite les témoignages de rescapés ou de témoins directs qui prennent le relais. Le récit est un mode de représentation qui se substitue à l'image manquante, sur un plateau le plus souvent vide, destiné à porter la parole dans toute sa nudité, ce qui la rend ainsi plus percutante. Dans Le Grand Silence, à la scène 7, cinq témoignages sont croisés pour reconstituer la mort des deux poètes :

Premier témoin

Malgré leurs supplications et leurs prières, nos camarades sont descendus de force dans un vallon voisin au bord d'un ruisseau. Pour préserver leurs vêtements, leurs bourreaux les déshabillent d’abord. Et bien sûr, dégainant leurs poignards, ils se jettent sur leurs victimes désarmées et sans protection. Sans pitié aucune, ils commencent à les transpercer et à les dépecer. Ils les amputent de leurs pieds et de leurs bras... Seul Daniel Varoujan tente de se défendre, et en guise de punition, les criminels, après avoir sorti ses viscères avec leurs couteaux, crèvent les yeux du poète (Le Grand silence, 1985, 45-46).

Le récit du massacre du poète est d'autant plus percutant que la scène précédente se terminait sur l'apologie des pouvoirs de la poésie et sur ces derniers mots de Daniel Varoujan : « Je pars avec l'image de l'Arménie dans mes prunelles. » L'effroyable nuit du 24 avril se termine dans un cri.

\section{LA REPRÉSENTATION DES BOURREAUX : ENTRE RECONSTITUTION HISTORIQUE ET FICTION}

Dans son théâtre du génocide, Pertj Zeytountsyan s'attaque à un tabou : la représentation du criminel politique, du génocidaire. Il faut songer que l'on se trouve en Arménie, face à un public fortement traumatisé par la non-reconnaissance et la négation du génocide: dans ce contexte, inscrire le bourreau dans l'espace scénique, lui donner vie, relève à la fois d'un défi (confronter le public au bourreau une nouvelle fois, et reproduire d'une certaine manière le trauma) et d'une nécessité (donner un visage à la barbarie pour pouvoir enfin la dépasser, sans pour autant rendre le bourreau trop humain, sans heurter la mémoire des disparus).

Pour cela, Pertj Zeytoutsyan fait du bourreau un être à la fois historicisé, pris dans l'Histoire, et un personnage de fiction, pris dans sa propre histoire. Si une partie des dialogues provient de sources documentaires, l'auteur prend la liberté de réinventer ses personnages pour en faire des archétypes du bourreau ordinaire, tel qu'il a été identifié par Hanna Arendt.

Sur la scène de Pertj Zeytountsyan, le bourreau se caractérise comme une figure hautement méprisable, souvent tenue à distance des autres personnages, comme s'il appartenait à une autre catégorie de l'humanité. Ses répliques recèlent un discours propagandiste marqué par la haine de l'Autre, identifié comme l'ennemi intérieur à éradiquer. L’Arménien est évoqué par le biais de métaphores avilissantes : Talaat Pacha, en réunion plénière de l'Ittihad déclare : «Les Arméniens sont une plaie 


\section{VIOLENCES RADICALES EN SCÈNE}

dangereuse pour notre corps. Au début, cette plaie empoisonnée n'est pas dangereuse, mais si elle n'est pas confiée à temps aux mains d'un chirurgien expérimenté, elle conduira à la mort. » (Debout! Le tribunal arrive, 1985, 65) Djemal Pacha utilise la métaphore animalière pour déshumaniser les victimes, des femmes affamées, assoiffées et sans défense, tout en les diabolisant:

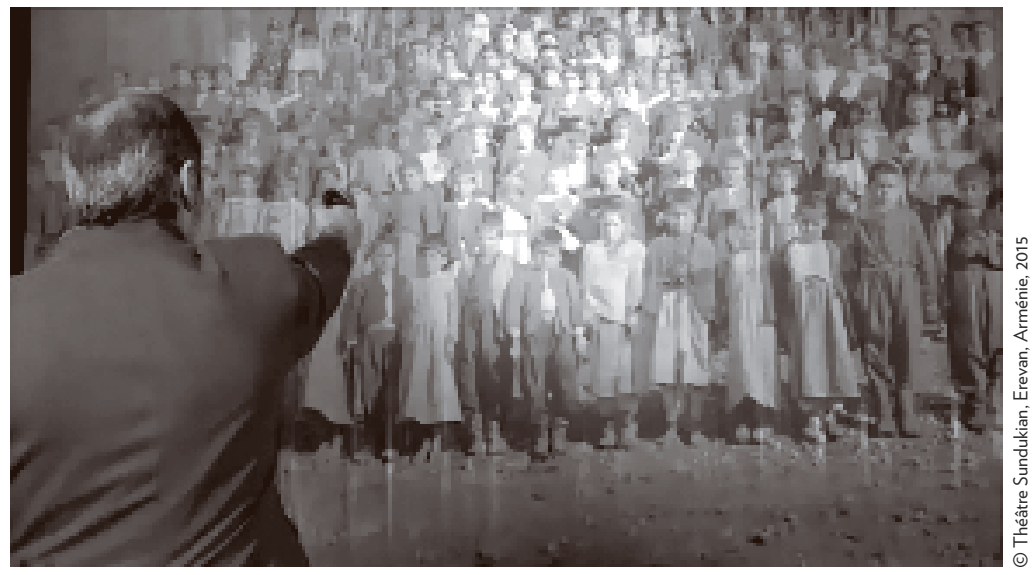

Vous pensez que je ne sais pas que vos maris se battent dans les forces armées d'Andranik, réplique-t-il à une vieille grand-mère squelettique qui l'implore, au nom des femmes déportées, de pouvoir rentrer chez elles. Vous voulez revenir chez vous pour avoir d'autres enfants qui vont après lutter contre nous. Les serpents n'ont pas de femelles et de mâles (Cent ans après, extrait de la vidéo de travail, 2015).

Le bourreau présente, bien entendu, des traits de sadisme, se délectant des carnages perpétrés dans tout l'Empire : alors que Daniel Varoujan agonise, au même moment, les massacres s'enchaînent à Erzindjian, à Kharpout, à Baïbourt, à Ourfa, à Bitlis, à Van, à Dyarbékir. Djemal Oghuz se livre sur scène à une comptabilité monstrueuse. Cependant, Pertj Zeytountsyan ne force pas le trait de la monstruosité outre mesure. Bien au contraire, le bourreau est avant tout homme comme les autres, tel Talaat, en train de se disputer avec sa femme qui tient absolument à ce qu'il mette ses pantoufles. S'il a des caractéristiques humaines, il n'a cependant aucune grandeur. Pertj Zeytoutsyan en fait un être veule, qui dans l'intimité révèle toute son impuissance. Face à Daniel Varoujan qui écrit sans relâche sous ses yeux, Djemal Oghuz a peur : «Tous les lâches craignent la poésie », conclut le poète. Pertj Zeytountsyan confie qu'il a voulu représenter le fait que « tous les bourreaux, quand ils perdent le pouvoir et vivent sous le poids des péchés, deviennent peureux et misérables » (Interview de Pertj Zeytountsyan réalisée par Robert Dermerguerian, 2006). L'auteur donne vie à ces figures de l'Histoire, en les démasquant, tout en les individualisant par des traits de caractère parfois ridicules, les saisissant dans des situations de la vie quotidienne presque dérisoires et inattendues. C'est ainsi que l'on voit Talaat, le sanguinaire, qui protège son petit moineau des griffes d'un chat. Par-là, Pertj Zeytountsyan évite l'écueil d’une représentation documentaire insoutenable (telle que Peter Weiss l'avait souhaitée par exemple pour la représentation des Procès d'Auschwitz dans L'Instruction). En même temps, ces traits spécifiquement humains accordés aux personnages permettent de poser la question des conditions dans lesquelles un être humain passe à l'acte et parvient non seulement à donner la mort, mais à ordonner une vaste boucherie organisée.
_ Djemal Pacha tire sur la photo des orphelins déportés rejouant de manière symbolique l'acte d'extermination sur scène. Cent ans après de Pertj Zeytountsyan, mise en scène de Tigran Gasparya. 
Le théâtre documentaire en Arménie

(suite)

\section{FICTION ET PARASITAGE DE LA SCÈNE}

L’ensemble du personnel dramatique des pièces de Petj Zeytountsyan est relié par un savant travail sur les jointures temporelles, notamment, par le biais de superpositions de temporalités induites par la filiation des personnages. Différentes figures issues du passé circulent dans le temps et parasitent l'espace scénique, en créant un hors-temps suspendu, celui d'une mémoire peuplée de fantômes. Cette atmosphère onirique qui se couple au montage documentaire est propre à l'esthétique de Pertj Zeytountsyan et permet de représenter visuellement les morts sans sépultures qui peuplent les déserts de Syrie-Mésopotamie, telles des âmes en peine et qui hantent les vivants. Cela peut se produire dans le moment de l'action, par une intrusion dans le temps historique. Dans Le Grand Silence, Pertj Zeytountsyan fait intervenir le personnage de Yerevoum, «l'Apparition » en arménien, qui, à la manière d'une représentation de la conscience collective nationale, ou d'un fantôme qui aurait déjà vécu le génocide, tente d'empêcher le massacre. Yerevoum distribue toutes ses pièces d'or aux gendarmes et aux Tchétés pour obtenir en échange la libération des deux poètes. Mais leur caravane s'en va. Daniel Varoujan et Ruben Sevak partent vers leur destin sans pouvoir entendre les cris de Yerevoum qui se débat contre le destin implacable de l'Histoire. Cette intrusion se produit généralement dans le temps vécu, comme dans Debout! Le tribunal arrive, lorsque Soghomon plaide non coupable lors du procès et fait le récit des événements dont il a été témoin:le metteur en scène utilise un système de trappes pour représenter la résurgence du passé et l'ouverture sur le monde des morts. La figure de la mère, qui hante Soghomon, est représentée sur scène, dans une posture à la fois noble, drapée d'un costume rouge sang qui la recouvre entièrement des pieds à la tête, et hagarde, perdue dans un hors-lieu et un hors-temps. Elle évolue en faisant des mouvements lents. La mise en scène réussit de cette façon à montrer la persistance des images obsédantes du passé dont les vivants ne peuvent se détacher tant que justice n'est pas rendue. Une figure semblable surgit sur la scène de Cent ans après, il s'agit de Mariam, la grand-mère d'Andranik. Mariam circule dans le temps, venant dans le présent, repartant dans le passé. Elle apparaitt, elle aussi, à son petit-fils lorsqu'il l'invoque, traduisant cette fois-ci sur scène, en plus, le traumatisme des générations suivantes qui se heurtent à une image générationnelle perturbée (la grand-mère morte plus jeune que ses descendants) et à l'impossible restitution de l'histoire des grands-parents tout comme de leur univers intérieur : "Vas-tu vieillir finalement! Mets une perruque ! Mais fais quelque chose! Tu ne m'as rien raconté de ta famille, de ton pays natal, de mes proches. » Parce qu'il arrive un moment où l'enquête historique ne parvient plus à combler les failles psychiques, Pertj Zeytountsyan utilise ces figures d'apparition pour restituer l'histoire vécue, pour rendre la parole à ceux qui ont été niés dans leur humanité. Face au fantôme de son bourreau, Djemal Pacha fait surgir sur scène, toute la cohorte des déportés, représentés à travers une photo qui remplit le fond de scène et qui fixe le temps du passé dans une sorte de permanence. Dans sa posture, les bras en croix, Mariam reproduit sur scène la crucifixion, sort qui a été réservé à 


\section{VIOLENCES RADICALES EN SCÈNE}

nombre de déportés, comme l'atteste son récit. Alors que Mariam est prosternée aux pieds de Djemal Pacha, celui-ci tire sur la photo des déportés, reproduit une nouvelle fois, dans le temps de la représentation, les tueries du passé, la pièce nous rappelle qu'en ne reconnaissant pas les faits, en n'écoutant pas les récits des rescapés, en les niant et en les réduisant au silence, on les assassine une deuxième fois.

Avec le théâtre de Pertj Zeytountsyan se produit, en définitive, un déplacement de la problématique. De la question de la représentation du génocide, l'auteur passe à celle du traumatisme à l'œuvre chez les rescapés et de son impossible dépassement si la qualification du crime n'existe pas (au moment du procès Téhléryan) et si la reconnaissance officielle n'a pas lieu (au moment de la rencontre avec Hassan Djemal). C'est ce qu'exprime de manière poignante le jeu de l'acteur qui interprète le rôle de Soghomon Téhléryan dans la mise en scène actuelle de Tigran Gasparyan. Effondré après son monologue final, Soghomon se met à frapper le sol avec une force inouïe. Il n'a en effet plus personne contre qui rediriger sa douleur, si ce n'est contre lui-même. La mise en scène révèle l'impossibilité pour le personnage de continuer à vivre malgré tout, car, même après la réparation symbolique que constitue la mort de Talaat et le verdict du procès, restent ces images d'une Histoire indélébile. Ces hommes, ces femmes, ces enfants qu'il évoque continuent à vivre en lui parce que, dit-il, « des gens comme eux ne peuvent pas mourir. » Après la demande de pardon d'Hassan Djemal, qui constitue « un premier pas » mémorable dans l'histoire des relations arméno-turques, dans Cent ans après, Armen reste seul, plus que jamais, face à ces un million et demi de morts : « fais un simple acte... compte de un jusqu’à un million et demi... sans arrêt, compte sans arrêt, et, peut-être, tu me comprendras définitivement. Et vous aussi, comptez... comptez... pour que certains d'entre vous n'osent plus jamais dire qu'il faut tourner la page. » Avec le théâtre de Pertj Zeytountsyan, on peut espérer la réconciliation, on peut croiser le bourreau ou serrer la main de son descendant, mais on ne peut oublier, surtout pas tant que la Turquie n'assumera pas son histoire. Les témoignages égrenés, les images obsessionnelles, les superpositions temporelles sont là pour nous le rappeler inlassablement.

\section{BIBLIOGRAPHIE}

- Lepsius, Johannes (1987) : Rapport secret sur les massacres d’Arménie (1915-1916) [1918], Paris, Payot.

- Zeytountsyan, Pertj (1985) : Debout ! Le Tribunal arrive (Votk'i Datarann é galis); Le Grand Silence (Medz Lerutjun) in Fuvres théâtrales (Tatron), Erevan, Sovietagan girq. Traduction réalisée par Annick Asso et Liliane Tchitchoyantz (2013), et publiée partiellement dans Annick Asso, Le Théâtre du Génocide, Paris, Honoré Champion, p. 209-223.

- Zeytountsyan, Pertj (2015) : Cent ans après, vidéo de travail produite par le Théâtre Sundukian, Erevan.

- Interviews de Pertj Zeytountsyan réalisées et traduites par Robert Dermerguerian en décembre 2006 et en septembre 2015. 